\title{
Optical In-Situ Measurement of Relative Deformations of the LHC Main Dipole Cold Masses
}

\author{
S. Aznar, J. Billan, J. Garcia Perez, M. La China, P. Ferracin, S. Redaelli, W. Scandale, and E. Todesco
}

\begin{abstract}
The LHC cryodipoles are composed of an evacuated cryostat and a cold mass, which is cooled by superfluid helium at 1.9 K. To obey constraints imposed by beam dynamics the particle beams must be centered within the mechanical axis of the dipole with a sub-millimeter accuracy. This requires in turn that the relative displacements between the cryostat and the cold mass must be monitored with accuracy at all times. Because of the extreme environmental conditions (the displacement must be measured in vacuum and between two points at a temperature difference of about 300 degrees), no adequate existing monitoring system was found for this application. We describe here a novel optical sensor developed for our scope and we present results of measurements made during the cold test of the dipoles.
\end{abstract}

Index Terms-Cold mass, displacement sensor, magnet alignment, superconducting magnet.

\section{INTRODUCTION}

$\mathbf{I}$ N THE Large Hadron Collider (LHC) under construction at CERN, two proton beams, circulating in opposite directions along the existing $27 \mathrm{~km}$ long tunnel built for LEP, should collide with a center of mass energy of $14 \mathrm{TeV}$, in four specially equipped experimental areas. Superconducting dipoles $15 \mathrm{~m}$ long with twin design producing the peak field of $8.36 \mathrm{~T}$ required in collision are used to guide the two counter-rotating beams along their nominal trajectory.

To make available the necessary mechanical aperture, i.e., the transverse clearance for circulating particles, with the minimum coil size, the active part of each dipole is bent in the horizontal plane and closely follows the beam trajectory. The dipolar field is entangled with unavoidable field-shape distortions, stemming from persistent current at injection field and from yoke saturation and coil deformations at collision field. To locally compensate their detrimental effect on beam stability, small multipolar correctors (sextupoles, octupoles and decapoles) are placed at the dipole ends. These features impose severe constraints on geometrical tolerance during dipole assembly. In each aperture, the maximum displacement between the beam reference orbit and the axis of the dipole cold bore should be rather small. The tolerance range is shaped as a torus of $1 \mathrm{~mm}$ radius in the bent part of the cold mass, and as a cylinder of $0.3 \mathrm{~mm}$ radius in

Manuscript received September 24, 2001.

S. Aznar, J. Billan, J. Garcia Perez, M. La China, P. Ferracin, W. Scandale, and E. Todesco are with the MMS Group, LHC Division, European Organization for Nuclear Research (CERN), 1211 Geneva 23, Switzerland (e-mail: \{sara.aznar; jaques.billan; juan.garcia.perez; marco.la.china; paolo.ferracin; walter.scandale; ezio.todesco\}@ cern.ch).

S. Redaelli is with the AP Group, SL Division, European Organization for Nuclear Research (CERN), 1211 Geneva 23, Switzerland (e-mail: stefano.redaelli@cern.ch).

Publisher Item Identifier S 1051-8223(02)04277-X. the straight ends [1]. In addition, the small multipolar correctors should be carefully centered on dipole axis and the dipole axis on the beam reference orbit to prevent the so-called harmonics feed-down effect inducing additional field-shape harmonics through relative misalignment. Systematic alignment errors should not exceed $0.3 \mathrm{~mm}$, whilst the standard deviation of random errors should be within $0.5 \mathrm{~mm}$ as discussed in [2]. With these constraints, the dipole assembly procedure becomes critical and must be assisted by survey measurements, based on laser trackers and optical moles [1]. The key point is to identify the center of the two cold bores in the bent cold mass and to reconstruct by a best-fit procedure the optimum path for the two beam orbits along each dipole. The subsequent operations required to complete the dipole assembly, i.e., the welding of the end-corrector supports, of the end-covers, of the support pads, of the interconnection bellows and of the bus-bar holders will use the virtual beam path as the reference for geometric shape. This is however insufficient to guarantee a safe beam operation. Indeed, the dipole should preserve its original shape also at the operational temperature $(1.9 \mathrm{~K})$. Furthermore, transient distortions of the cold mass, eventually induced by thermal gradients during cool down and warm-up, should be compatible with the mechanical tolerance of interconnection bellows, which can stand occasional transverse deformations of $4 \mathrm{~mm}$ at most.

To investigate these issues we developed, in collaboration with the EPFL (École Polytechnique Fédérale de Lausanne, $\mathrm{CH}$ ) and industry (Smartec S.A., Lugano, $\mathrm{CH}$ ), an optical detector, based on low-coherency interferometry, to measure in-situ the dipole cold mass deformation relative to the cryostat during thermal and powering cycles [3], [4]. The detector has been installed in four dipoles, three of which have been tested in cold conditions. In this paper we present data collected during these tests. In Section II we describe the concepts on which our detector is based. In Section III, we show how we adapted it to measure the distortion of the LHC dipole respect to the cryostat. In Section IV we present and discuss our experimental results. Finally, in Section V, we draw some conclusions.

\section{Technology of the OpticAl Detector}

The detector used in the LHC cryo-dipole is derived from a monitoring system, called SOFO (Surveillance d'Ouvrages par Fiber Optique), originally developed to observe deformations of civil-engineering structures [5]. The SOFO sensor, schematically shown in Fig. 1, is a low-coherency double interferometer in tandem configuration, detecting the length difference between a measurement fiber attached to the host structure under inspection and a reference fiber freely installed, unaffected by the structure displacements. 


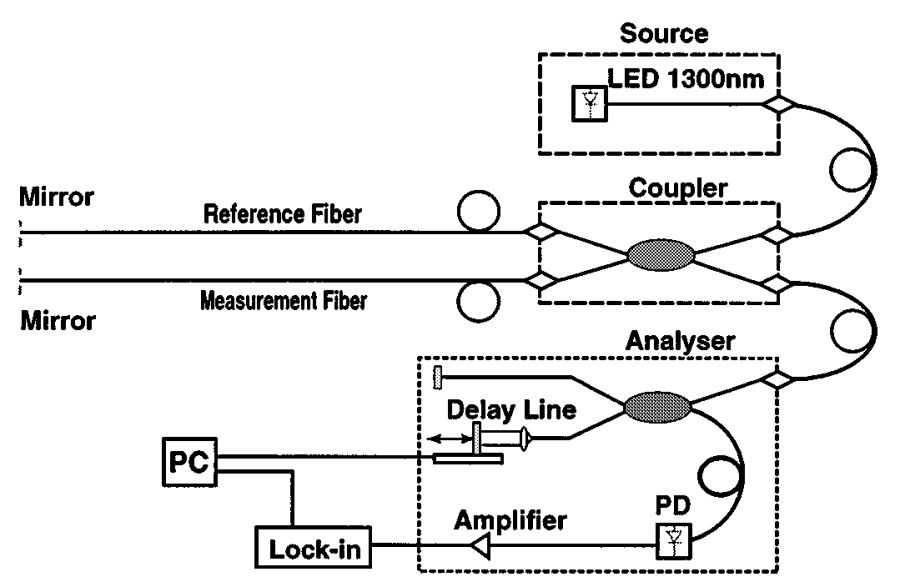

Fig. 1. Scheme of the SOFO detector.

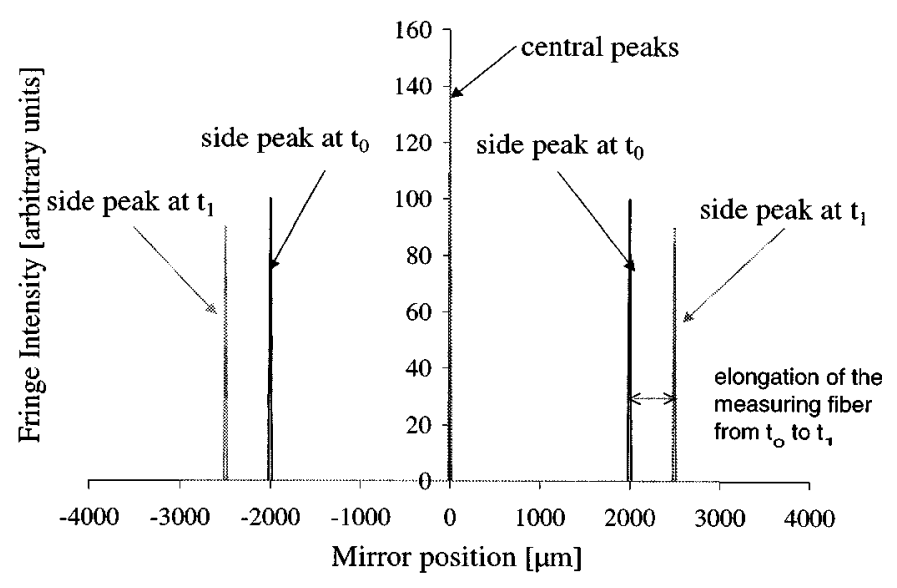

Fig. 2. Fringe intensity as a function of the mirror position.

The light emitted by a diode (LED) operating at $1.3 \mu \mathrm{m}$ with a coherency length of $30 \mu \mathrm{m}$, launched through a coupler in the two fibers and reflected back by reflecting ends, is recombined and produces fringes due to the difference of optical paths. The second Michelson interferometer allows measuring the path unbalance, using also two fibers and a delay line made of a mobile mirror. On moving the mirror, the light intensity irradiating the photodiode (PD) produces a triple coherence peak. The central peak appears when the two fibers in the analyzer arm have the same length and the side peaks appear when the path unbalance in the analyzer arm corresponds to the absolute length difference of the two fibers in the measuring arm. By following the position of the side peaks, as shown in Fig. 2, it is possible to determine the total elongation undergone by the measurement fiber and by the mechanical structure under inspection, with a typical precision of about $2 \mu \mathrm{m}$.

\section{AdAptation of the Detector to the LHC Dipole}

The SOFO sensor is in principle adequate to measure transverse deformations of the dipole shape during LHC operation. However it cannot directly serve to this scope, since it is difficult to integrate a measuring optical fiber on the cryodipole. Indeed, the large temperature gradient between the cold mass $(1.9 \mathrm{~K})$ and the cryostat vessel $(300 \mathrm{~K})$, suggests preventing thermal

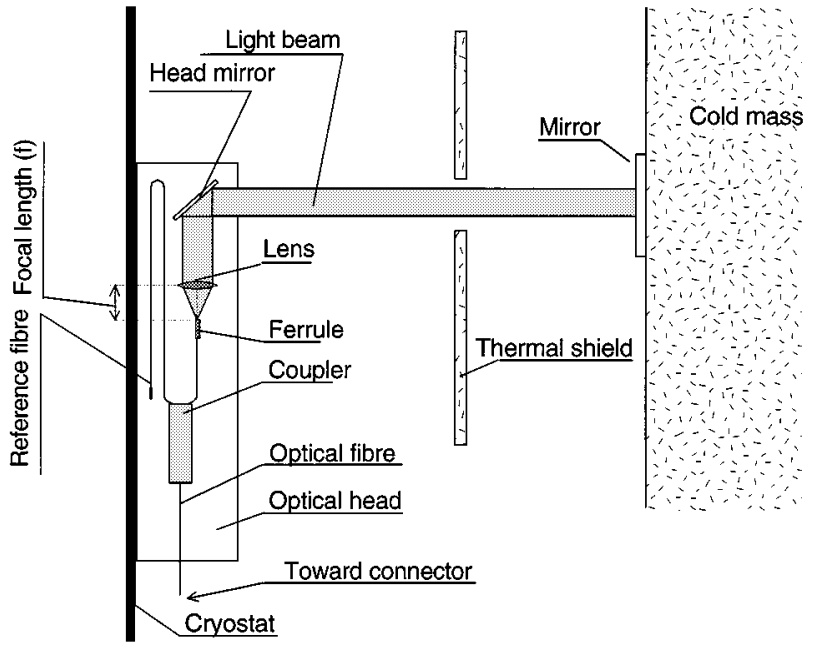

Fig. 3. Double-pass delay line.

contacts even through glass fibers. Furthermore, the longitudinal contraction of about $40 \mathrm{~mm}$ of the cold mass during cool down eventually entangles the effects of longitudinal and transverse displacements making interpreting the experimental data difficult. To overcome these problems, we used the double pass delay line in Fig. 3 as a measurement line: a mirror placed on the cold mass reflects the radiation emitted by an optical fiber through an optical head, located on the inner wall of the vacuum vessel. The LED primary radiation enters in the vessel through a fiber and an optical connector, illuminating through a coupler both the reference fiber and the delay line. In the latter, the fiber end, mounted inside a polished reflective ferule, irradiates a focusing lens and the head and the cold mirrors. The light is reflected back and forth from the cold mirror to the ferule. As the position of the Fiber end and the lens focal point are optically conjugated through the lens-mirrors system after two round trips (double pass delay line), the light will be focused back to the fiber core. The coupler mixes this radiation with the light reflected back by the reference Fiber end and feeds a SOFO-type analyzer arm monitoring the distance between the ferule and the mirror, i.e., between the vessel and the cold mass. A critical point is the orientation of the mirror, which can change during thermal cycles and dipole operation. We chose optical components providing \pm 5 mrad tilt acceptance. We installed three sensors per dipole end, two along a horizontal diameter and one in the lower part of the cold mass, along the vertical axis. They provide information on the diameter size and on the horizontal and vertical positions of the cold mass center.

\section{EXPERIMENTAL DATA}

\section{A. Available Data}

We collected data during thermal and powering cycles, and occasionally after quenches. In each instrumented dipole end, we measured, whenever possible, two horizontal and one vertical distances between the cold mass and the cryostat. Using them we computed the variations of the cold mass diameter and the horizontal and vertical displacements of the center. 


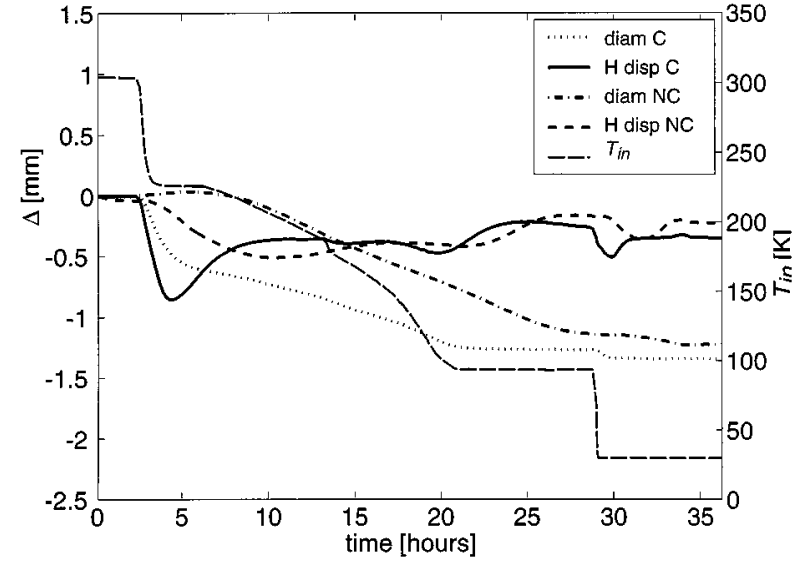

Fig. 4. Cool down of the prototype MBP2A2. Horizontal shift.

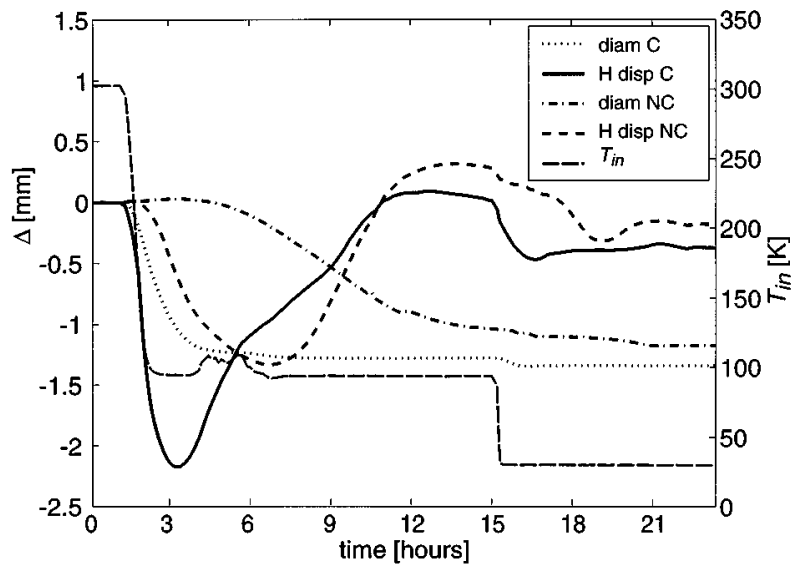

Fig. 5. Fast cool down of the prototype MBP2A2. Horizontal shift.

\section{B. Thermal Cycles}

In Fig. 4, we present data collected during a cool down at nominal rate of the prototype dipole MBP2A2. We show the time evolution of the temperature $T_{\text {in }}$ (right scale) as measured on the cold mass vessel close to the connection side, where is the inlet of the cooling helium. We also show the evolution of the diameter and the horizontal position of the center (left scale), both at the connection (C) and nonconnection (NC) dipole ends. Positive displacements of the center are outwards with respect to the center of the cold mass curvature.

From 300 to $1.9 \mathrm{~K}$, the dipole diameter is expected to shrink of about $1.35 \mathrm{~mm}$. The observed change was always very close to this value, thereby confirming the correct operation of our detector and the validity of the collected data. After a temperature step, the C-side diameter shrinks immediately whilst the diameter of the NC-side, which is reached later by the cooling helium, starts shrinking after a few hours. During this period, there is temperature gradient along the dipole. Each temperature step induces initially a shift of the dipole center toward the center of curvature of the dipole (negative shift). Then the center tends to recover the original position. The $\mathrm{C}$-center is deformed immediately and the NC-center after a few hours.

Fig. 5 shows what happens in the dipole MBP2A2 during a fast cool down, i.e., when helium at the liquid nitrogen temperature is directly injected in the dipole. The initial temperature step

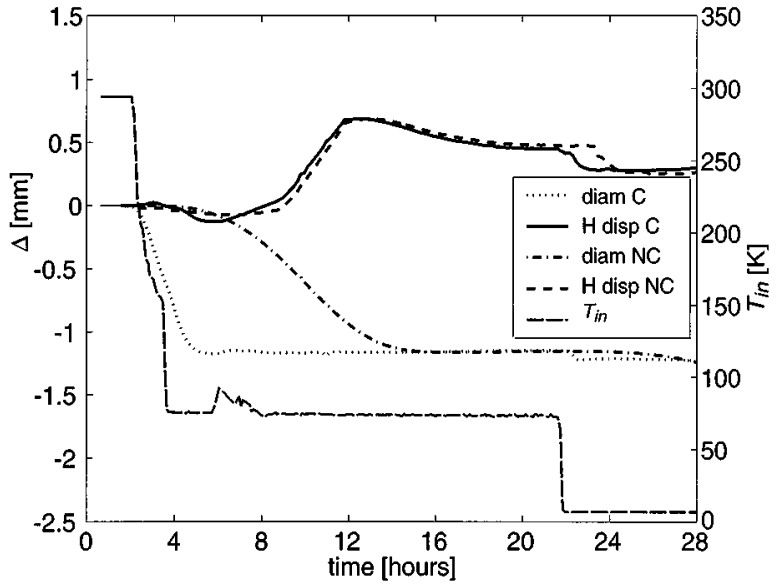

Fig. 6. Fast cool down of the dipole HCMBB_A001-01 000001 . Horizontal shift.

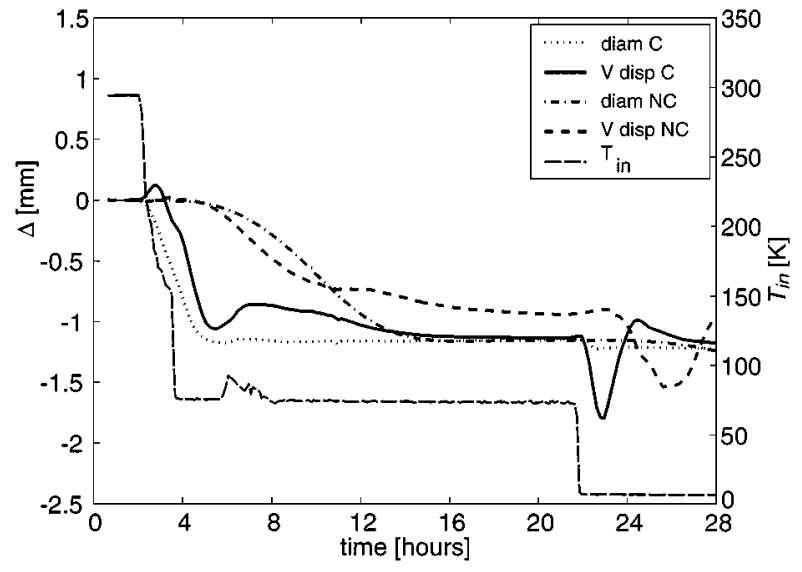

Fig. 7. Fast cool down of the dipole HCMBB_A001-01 000 001. Vertical shift.

is of about $200 \mathrm{~K}$. The evolution of the observed phenomena is qualitatively the same as for a nominal cool down, except that the shift of the center is now larger by more than a factor of 2 . In each dipole, the thermal shift is always roughly proportional to the initial step of temperature, however the proportionality coefficient varies considerably from dipole to dipole.

In Figs. 6 and 7, we show the effect of a fast cool down in the pre-series dipole HCMBB_A001-01000001, respectively in the horizontal and the vertical directions. The changes of the cold mass diameters in the $\mathrm{C}$-side and $\mathrm{NC}$-side are very similar to those of MBP2A2 dipole. However, the horizontal position of the centers varies much less. In the given example, the negative variation induced by the initial temperature step is of about $-0.2 \mathrm{~mm}$ instead of $-2.2 \mathrm{~mm}$ (see Figs. 5 and 6). On the other hand, the later positive variation is of about $1 \mathrm{~mm}$. Other observations made both in prototypes and pre-series dipoles show that for the same temperature gradient, the centers of the preseries dipoles are deformed in the horizontal direction three to four times less in comparison with the prototype dipoles.

The vertical shift (see Fig. 7), mainly induced by the shrinking of the feet and of the cold mass, features the following pattern. After a temperature step, the center descends and then comes up again. On the other hand, the $\mathrm{C}$-side is affected immediately and the NC-side is deformed a few hours 
TABLE I

Shift of the Dipole Center $\Delta C$ InduCed by Thermal CyCle. The AvERAGE $\pm 1 \bullet \sigma$ OVER $n$ DATA AVAILABLE IS Given

\begin{tabular}{c|c|c|c|c|c}
\hline \multirow{2}{*}{$\begin{array}{c}\text { OBSERVATION } \\
\text { DIRECTION }\end{array}$} & \multicolumn{2}{|c|}{ NC-SIDE } & \multicolumn{2}{c}{ C-SIDE } \\
\cline { 3 - 6 } & $n$ & $\Delta C[\mathrm{~mm}]$ & $n$ & $\Delta C[\mathrm{~mm}]$ \\
\hline \multirow{2}{*}{ COOL } & HORIZONTAL & 7 & $-0.08 \pm 0.14$ & 3 & $-0.14 \pm 0.38$ \\
\cline { 2 - 6 } DOWN & VERTICAL & 3 & $-0.87 \pm 0.13$ & 3 & $-1.03 \pm 0.24$ \\
\hline WARM & HORIZONTAL & 3 & $0.15 \pm 0.13$ & 1 & 0.10 \\
\cline { 2 - 6 } UP & VERTICAL & 1 & 1.00 & 1 & 0.85 \\
\hline
\end{tabular}

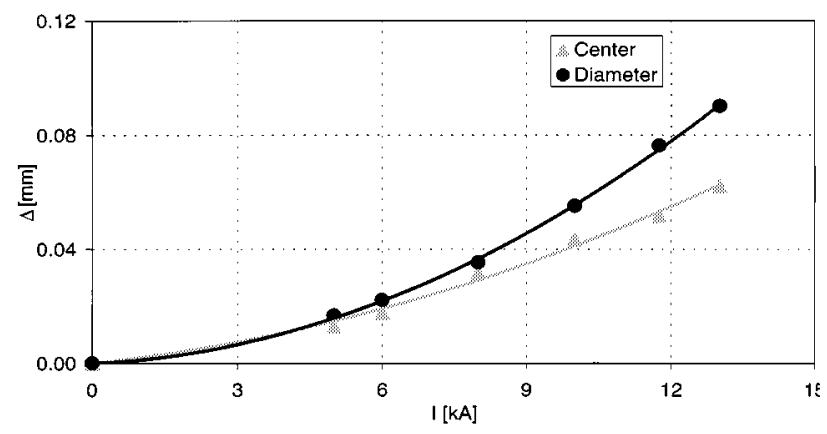

Fig. 8. Effect of the energization of the prototype MBP2O1.

later. This behavior is also visible after the thermal step at $100 \mathrm{~K}$. It should be mentioned that the synchronism of the horizontal deformations observed in the $\mathrm{C}$-side and $\mathrm{NC}$-side and shown in Fig. 6 is at present unexplained and should be confirmed by further measurements.

\section{Change of Shape From Room to Cryogenic Temperature}

It is important to check if there is any shift of the dipole ends, induced by the temperature variation from $300 \mathrm{~K}$ to $1.9 \mathrm{~K}$ and vice versa. We made several observations during cold down and warm up, using nominal or fast cycles, both on prototypes and pre-series dipoles. The data available are homogeneous for all dipole types and thermal cycle speeds. Shifts measured at the end of thermal transient are given in Table I.

Average values and standard deviations of the cold mass center displacement $(\Delta C)$ are computed over the number of available observations $n$. Shifts measured in NC-side and $\mathrm{C}$-side ends are the same within the statistical error in both directions. The average effect of warm up and cool down features opposite signs in the two directions. In the horizontal direction, the observed shift is tolerable for beam stability. In the vertical direction, however, the average dipole shift is to be compared with the average quadrupole shift, to assess the stability of the beam orbit (mostly determined by quadrupole positioning) with respect to the position of the correctors attached at the dipole ends.

\section{Effect of Powering Cycle}

During powering cycles, the electromagnetic force in the dipole coil increases with the square of the exciting current. At the nominal current of $11.75 \mathrm{kA}$, the outward horizontal force per unit length in the cold mass cross section is about $3 \mathrm{MNm}^{-1}$, whilst the outwards longitudinal force at the dipole ends is about 0.5 MN [4]. This determines the increase of the horizontal diameter and the straightening of the dipole. In Fig. 8 we show this effect as observed in the dipole MPB2O1.

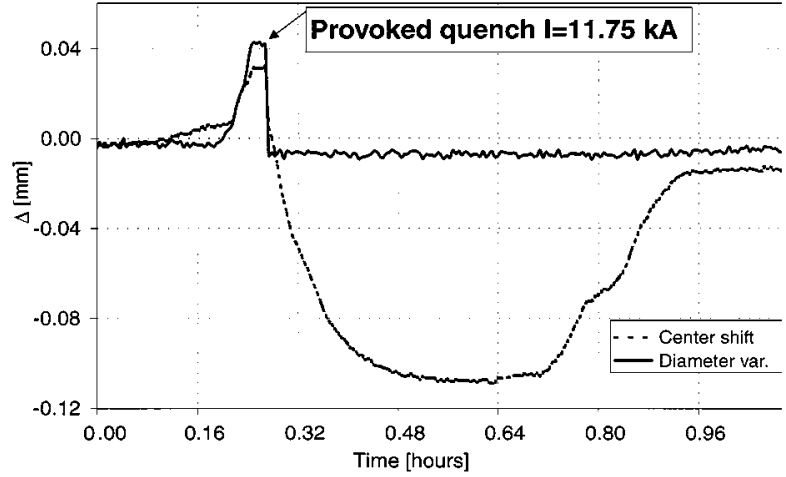

Fig. 9. Effect of quench in the prototype MBP2O1.

The diameter increases up to $80 \mu \mathrm{m}$ and the center is shifted up to $60 \mu \mathrm{m}$. Both of them feature a quadratic dependence with the excitation current. The measured diameter change is close to numerical predictions.

\section{E. Effect of Quench}

During quenches, the nominal energy of about $7 \cdot 10^{6} \mathrm{~J}$ stored in a dipole is partly dissipated inside the coils with a consequent increase of the average temperature of the cold mass up to $30 \mathrm{~K}$. The electromagnetic forces induced by current disappear as well and the dipole suddenly recovers its original diameter and position. In these conditions, the cooling fluid starts removing the thermal energy from the cold mass, inducing again distortion of the dipole ends. The diameter does not move since at these temperatures thermal variations are negligible. In Fig. 9 we show an example of this phenomenon. The time evolution of the diameter and the horizontal position of the center are shown during a quench which occurred at $11.5 \mathrm{kA}$ in the dipole MBP2O1. By analyzing quenches at different currents we saw that the dipole recovers its temperature of $1.9 \mathrm{~K}$ and hence its pre-quench shape with a time constant ranging from 20 to 50 minutes.

\section{CONCLUSIONS}

We described an optical device detecting in-situ distances between the LHC cold mass and its cryostat, with a precision of $0.01 \mathrm{~mm}$. We observed deformations of the dipole ends induced by thermal cycles of a few tenth of millimeters in horizontal direction and of $1 \mathrm{~mm}$ in the vertical one. The latter should be compared in the future to the displacement of the quadrupole cold mass to confirm the mutual alignment of dipoles and quadrupoles in operational condition. During thermal transients we also observed large transient displacements of the dipole ends, which have no detrimental effects on beam dynamics but may induce undesired stresses to the interconnecting bellows. We finally measured very tiny effects induced by the powering cycle and by quenching, confirming the high quality and sensitivity of the detector. To observe more realistic situation our sensor will be mounted in the dipoles of the LHC test cell.

\section{ACKNOWLEDGMENT}

The authors would like to acknowledge C. Wyss for the constant support to this project. B. Glisic and D. Inaudi of Smartec SA and S. Fakra of the EPFL took part in the development of 
their sensor. M. Bajko and A. Pardons helped the authors in the installation and the alignment of the optical components. The authors also acknowledge the MTA and CRI Group of the CERN-LHC Division.

\section{REFERENCES}

[1] W. Scandale, M. Bajko, and F. Savary, "Alignment and geometry requirements for the LHC main dipole," in 7th European Particle Accelerator Conf., 2000, pp. 2160-2163.
[2] R. Bartolini and W. Scandale, "Multipole feed-down due to dipole misalignments,", CERN LHC MMS Internal Note 97-12, 1997.

[3] D. Inaudi, B. Glisic, S. Fakra, J. Billan, J. Garcia Perez, S. Redaelli, and W. Scandale, "Development of displacement sensor for the CERN-LHC superconducting cryodipoles," Meas. Sci. Technol., vol. 12, 2001.

[4] S. Redaelli, "Analysis of the magnetic field perturbations in dipoles and quadrupoles of the large hadron collider (LHC)," Diploma Thesis, Milano, 2000.

[5] D. Inaudi, "Fiber optic sensor network for the monitoring of civil structures," EPFL Ph.D. Thesis 1612, Lausanne, CH, 1997. 